Vari abi l i ty i $n$ tei copl ani $n$ protei $n$ bi ndi ng and i ts predi cti on usi ng serumal bumin concent $r$ at $i$ ons

\begin{tabular}{|l|l|}
\hline 著者 & $\begin{array}{l}\text { YANO Ryoui chi, NAKAMRA Toshi aki, TSUKAMOTO } \\
\text { H t oshi, I GARASH Toshi aki, GOTO Nobuy uki , } \\
\text { WAK YA Yoshi f um, MASADA M ki o }\end{array}$ \\
\hline $\begin{array}{l}\text { j our nal or } \\
\text { publ i cat i on t i t l e }\end{array}$ & Ther apeut i c dr ug moni t or i ng \\
\hline vol une & 29 \\
\hline number & 4 \\
\hline page r ange & $399-403$ \\
\hline year & $2007-08$ \\
\hline URL & ht t p: //hdl . handl e. net /10098/1935 \\
\hline
\end{tabular}




\title{
Variability in Teicoplanin Protein Binding And Its Prediction Using Serum Albumin Concentrations
}

\author{
Ryoichi Yano, MS Toshiaki Nakamura, MS Hitoshi Tsukamoto, BS \\ Toshiaki Igarashi, BS Nobuyuki Goto, $\mathrm{PhD} \quad$ Yoshifumi Wakiya, $\mathrm{PhD}$ \\ Mikio Masada, $\mathrm{PhD}$
}

Department of Pharmacy, University of Fukui Hospital

Address correspondence and reprint requests to Ryoichi Yano, MS.,

Department of Pharmacy, University of Fukui Hospital, 23 Matsuokashimoaizuki,

Eiheiji, Fukui 910-1193 JAPAN

(E-mail: yanor@fmsrsa.fukui-med.ac.jp)

Phone: +776-61-3111

Fax: $+776-61-8256$

Sources of support: We don't have any financial supports that require acknowledge. 


\begin{abstract}
The impact of lower serum albumin levels on teicoplanin pharmacokinetics has not been previously determined. The authors assessed the relationship between total and free concentrations of teicoplanin in serum samples obtained from patients receiving teicoplanin therapy for gram-positive bacterial infections. In addition, the authors determined the contribution of serum albumin concentrations to the unbound fraction of teicoplanin.
\end{abstract}

One hundred ninety-eight serum samples were obtained from 65 patients undergoing routine therapeutic drug monitoring of teicoplanin. Free serum teicoplanin was separated by ultrafiltration, and total and free serum concentrations of teicoplanin were determined by a fluorescence polarization immunoassay. Regression analysis was then performed to build a prediction model for the free serum teicoplanin concentration from the total serum teicoplanin concentration and the serum albumin level using the first 132 samples. The predictive performance of this model was then tested using the next 66 samples.

Free serum teicoplanin concentrations $(C f)(\mathrm{\mu g} / \mathrm{mL})$ were predicted using a simple model constructed using total serum teicoplanin $(C t)(\mu \mathrm{g} / \mathrm{mL})$ and albumin concentrations 
$(A L B)(\mathrm{g} / \mathrm{dL}): C f=C t /(1+1.78 * A L B)$. This model could estimate free serum teicoplanin concentrations with a small bias and an acceptable error. The measured free level of teicoplanin will lie between 0.63 and 1.38 times the predicted concentration in $95 \%$ of cases.

Serum albumin level plays a major role in the variability of the fraction unbound of teicoplanin. This model can reliably estimate free serum teicoplanin concentrations more easily than by using direct measurements.

Keywords: teicoplanin, fraction unbound, albumin, pharmacokinetics 


\section{Introduction}

Recent reports have recommended that therapeutic drug monitoring be used to maintain an adequate serum trough levels when teicoplanin is administered to patients with severe infections ${ }^{1,2}$. In Japan, therapeutic drug monitoring is therefore recommended to achieve suitable teicoplanin trough total concentrations $(>10 \mu \mathrm{g} / \mathrm{mL}$ for severe infections) for each patient ${ }^{3}$.

Methicillin-resistant Staphylococcus aureus (MRSA) is an organism that causes hospital infections in many countries ${ }^{4,5}$. Teicoplanin and vancomycin are effective drugs for the treatment of infections caused by gram-positive cocci, including MRSA ${ }^{6}$. Recently, however, strains that show lower susceptibility to vancomycin has been reported, and the appropriate usage of glycopeptides is strongly recommended ${ }^{7,8}$. Of cause, limiting the administration of antibiotics to minimize adaptation is an important concern, but also optimizing the dosage regimen for individual patients is also needed to achieve sufficient serum concentrations ${ }^{9}$. Recent studies suggest that teicoplanin exhibits time-dependent killing ${ }^{10}$, and serum trough levels are an important factor in the clinical outcome $e^{11}$.

The pharmacokinetics of teicoplanin has been studied and is known to vary under 
differing conditions, including renal impairment ${ }^{12}$, renal support therapies ${ }^{13}$, elderly ${ }^{14}$, and neutropenia ${ }^{15}$. MRSA infections may occur frequently on such immunocompromised hosts. In such patients, lower serum albumin concentrations are often observed ${ }^{16,17}$.

Teicoplanin is known to bind strongly to serum albumin. From in vitro studies, it is clear that protein binding affects bacterial killing with teicoplanin ${ }^{18}$. Bernareggi et al. reported that the binding of teicoplanin to plasma protein is linear up to about $300 \mathrm{mg} / \mathrm{L}$ and the fraction unbound (fu) value is not dose-dependent between 15 and $25 \mathrm{mg} / \mathrm{kg}$ dose ${ }^{19}$. The effect of changing serum albumin levels on the fraction unbound of teicoplanin, however, was not determined.

We assessed the relationship between total and free concentrations of teicoplanin in serum samples obtained from patients undergoing routine therapeutic drug monitoring. We also determined the contribution of serum albumin concentrations on the fraction unbound of teicoplanin. 


\section{Materials and Methods}

\section{Subjects}

Between October 1998 and May 2002, 198 serum samples were obtained from the routine therapeutic drug monitoring of 68 patients receiving teicoplanin therapy to treat gram-positive infections at member hospitals of the Fukui Therapeutic Drug Monitoring Research Group. The patients included 43 men and 24 women and had a median age of 72years. Median serum creatinine and albumin concentrations prior to teicoplanin administration were $0.7 \mathrm{mg} / \mathrm{dL}$ and $3.2 \mathrm{~g} / \mathrm{dL}$, respectively.

\section{Ethics Approval}

In University of Fukui hospital, written consents about routine investigations using clinically collected data have been obtained from patients. In other hospitals, research use of serum samples for this study was approved by an appropriate organization of each institute.

\section{Determination of Serum Total and Unbound Serum Teicoplanin}

Serum samples were stored at $-20{ }^{\circ} \mathrm{C}$ until analysis. Unbound teicoplanin was separated by ultrafiltration using Centrifree ${ }^{\circledR}$ YM-30 devices (Nihon Millipore, Tokyo, Japan). Serum teicoplanin concentrations were assayed by a fluorescence polarization 
immunoassay using a INNOFLUOR ${ }^{\circledR}$ Teicoplanin Assay System (Seradyn, Indianapolis, IL, U.S.A) with the TDxFLx system (Abbott Laboratories, Chicago, IL, USA). To assay the free serum teicoplanin, certain parameters of the TDxFLx system were changed to quantify a lower concentration range.

\section{Determination of Serum Albumin Concentrations}

Serum albumin concentrations were measured by colorimetric detection using bromocresol purple ${ }^{20}$ on an automated analyzer (Nipro, Tokyo, Japan).

\section{Model Development and Validation}

We collected data sets that included total serum teicoplanin concentrations (TEIC total $)$, free serum teicoplanin concentrations $\left(\mathrm{TEIC}_{\text {free }}\right)$, and serum albumin concentrations (ALB). The fraction unbound ( $f u$ ) of teicoplanin was calculated as follows:

$f u=\mathrm{TEIC}_{\text {free }} / \mathrm{TEIC}_{\text {total }}$

These data were separated into two groups according to the time period in which the samples were gathered. Of the 198 samples, the first 132 samples were used for the model development and the final 66 samples were used to validate the model.

The parameter characterizing the protein binding of teicoplanin ( $\mathrm{nKa}$ ) was estimated using the model development data set by least square regression analysis according to 
the equation below, which is derived from the Scatchard equation:

$\mathrm{TEIC}_{\text {free }}=\mathrm{TEIC}_{\text {total }} /(1+\mathrm{nKa} * \mathrm{ALB})$

The value of $\mathrm{nKa}$ was the product of the number of binding sites on albumin and the association constant.

To validate this model, the predictive ability for $\mathrm{TEIC}_{\text {free }}$ was tested using the model validation data set. TEIC total, $\mathrm{ALB}$, and the value of $\mathrm{nKa}$ were used to calculate $f u$ and determine TEICfree. Predicted and measured TEIC free were analyzed by Altman-Bland difference-style plot ${ }^{21}$. A $p$ value less than 0.05 was considered to be significant. Data and statistical analysis were performed using Kyplot software (version 3.0. Kyence, Tokyo, Japan). 


\section{Results}

\section{Assay Validation}

The accuracy of the TEIC free assay at concentrations of $0.5,3.0$ and $5.0 \mu \mathrm{g} / \mathrm{mL}$ was within the ranges of $102-107 \%$. The coefficients of variation (CV) relative to the three concentrations of the TEIC free assay in the within- and between-run studies were both less than $5 \%$.

For the TEICtotal assay, all $\mathrm{CVs}_{\mathrm{s}}$ in the within- and between-run studies using commercially available control samples were less than $9 \%$.

\section{Sample Characteristics}

The patient characteristics are shown in Table 1. The model-development group exhibits significant differences from the model validation group; for example, the median serum albumin levels were lower in the model validation group than in the model development group. There are no differences on patients' age, body weight, and kidney function in the two patient groups. 


\section{Serum Albumin Concentration and the Fraction Unbound of Teicoplanin}

The relationship between serum albumin concentration and the fraction unbound of teicoplanin is shown in Figure 1. A marked increase in the fraction unbound was observed concurrent with a decrease in the serum albumin levels.

\section{Intra- and Inter- patients variability of the fraction unbound of teicoplanin}

There are large intra-patients variations of the fraction unbound of teicoplanin in some patients. The most considerable case, the fraction unbound of teicoplanin was changed 0.06 to 0.19 in 2 weeks. Inter-patients variability of the fraction unbound of teicoplanin was determined by calculating the coefficient variation (CV) of the patient's mean of the fraction unbound of teicoplanin and yielded $28.8 \%$.

\section{Model Development and Validation}

$\mathrm{TEIC}_{\text {free }}$ as a function of $\mathrm{TEIC}_{\text {total }}$ and $\mathrm{ALB}$ is illustrated in Figure 2. The value of nKa ( \pm standard error) was $1.78( \pm 0.03)(\mathrm{g} / \mathrm{dL})^{-1}$. Using this model, TEIC free was predicted by the model validation data set. The difference plot for predicted and measured values of

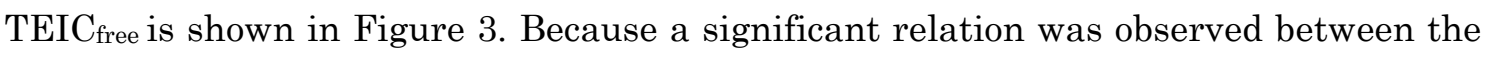
difference and the mean $(r=0.31, p=0.011)$, data were analyzed by log transformed. The mean difference is $-0.029 \mathrm{\mu g} / \mathrm{mL}$ and limits of agreement (95\% confidence interval) are -0.20 and 0.14 on the log scale, respectively. Taking the antilog of these limits, it means 
that the measured free level of teicoplanin will lie between 0.63 and 1.38 times the predicted concentration in $95 \%$ of cases. 


\section{Discussion}

In an in vitro study, the killing activity of teicoplanin was affected by the presence of serum ultrafiltrate ${ }^{18}$. This supports the hypothesis that the high degree of protein binding may be a factor that affects the killing activity of teicoplanin. From a pharmacokinetic theory standpoint, it is clear that only free drugs can pass through microvessels and the cell membranes to reach pharmacologic targets (i.e. infection sites or pathogenic organs). We should consider free drug concentrations to understand delivery and bactericidal activities during infectious disease treatment. However, only a small number of reports have discussed free teicoplanin concentrations in clinical settings with regard to its strong protein binding capability. Recently, the relationship between free steady-state trough teicoplanin concentrations and epithelial lining fluid concentrations in patients with ventilator-associated pneumonia was reported ${ }^{22}$. In this paper, the authors reported that free steady-state trough teicoplanin concentrations were comparable to concentrations of epithelial lining fluid. They also suggested that the fraction unbound of teicoplanin was much higher than expected due to lower levels of serum albumin.

Though further study is needed to confirm the impact of free serum concentrations of teicoplanin on its pharmacological effects and adverse drug reactions, technical 
difficulties and complexities related to the monitoring of free serum teicoplanin concentrations hinder research in this field. In our study, we developed an improved FPIA method to measure lower concentrations of teicoplanin. Our method could accurately quantify $0.5 \mu \mathrm{g} / \mathrm{mL}$ of teicoplanin in serum filtrate samples. We think that the routine analysis of free teicoplanin concentrations is not necessary. Thus, these methods of determining free teicoplanin concentrations may help develop a better understanding of the pharmacokinetic-pharmacodynamic relationships of teicoplanin.

Our model, presented in Figure 2, could estimate free serum teicoplanin concentrations with a small bias and a slightly large but an acceptable error using total teicoplanin concentrations and albumin levels. From the analysis represented in figure 3 , the limits of agreement are -0.20 and 0.14 on the log scale. This means that for about 95\% of cases the result of $\mathrm{TEIC}_{\text {free }}$ prediction will be between 0.63 and 1.38 times the measured TEICfree.

Renal dysfunction sometimes accompanies hypoalbuminemia, and some reports have indicated that the accumulation of endogenous ligands affects phenytoin binding to a greater extent than serum albumin concentrations ${ }^{23,24}$. Based on multiple variable analyses, serum urea nitrogen and total bilirubin are not variables that influenced teicoplanin protein binding (data not shown). Few uremic patients were included in our 
study, so our results only applicable to the patients who have a similar degree of the renal function as presented in Table1.

Figure 4 is a useful nomogram for the prediction of the free serum concentrations of teicoplanin, and was created by calculations at various serum levels using the model equation presented in Figure 2. To estimate the free serum teicoplanin concentration, only the levels of serum albumin and total teicoplanin are required. Significant variability is present in the free teicoplanin fraction both within and between patients. During antimicrobial chemotherapy, it is very important to achieve sufficient drug exposure to pathogenic organisms. The variability observed in our patients, however, suggests that the true exposure might be different even if the same total teicoplanin concentrations were obtained. In patients with hypoalbuminemia, a variation of fraction unbound of teicoplanin should be considered to avoid unnecessary and costly over dosing. Considerable effects of decreased serum albumin levels on the fraction unbound of teicoplanin were apparent when serum albumin levels were below $3.0 \mathrm{~g} / \mathrm{dL}$ (Fig. 1). This indicates that only for patients with hypoalbuminemia might it be necessary to measure the fluctuation of the fraction unbound of teicoplanin.

Here, we present a clear contribution of serum albumin to the fraction unbound of teicoplanin, and also provide a simple model that can predict the free concentration of 
teicoplanin based on the total concentration of teicoplanin. 


\section{Acknowledgements}

The authors gratefully acknowledge the efforts of our pharmacy staff for excellent

technical support. 


\section{References}

1 MacGowan AP. Pharmacodynamics, pharmacokinetics, and therapeutic drug monitoring of glycopeptides. Ther Drug Monit. 1998; 20: 473-477.

2 Ueda Y, Shibata Y, Ogawa F, et al. Clinical efficacy of teicoplanin against MRSA infections in emergency and critical care medicine. Nippon Kagaku Ryoho Gakkai Zasshi. 2003; 51: 490-496.

${ }^{3}$ Targosid ${ }^{\circledR}$ Package insert in Japan.

4 Tiemersma EW, Bronzwaer SL, Lyytikainen O, et al. Methicillin-resistant Staphylococcus aureus in Europe, 1999-2002. Emerg Infect Dis. 2004; 10: 1627-1634

${ }^{5}$ Kobayashi H. National hospital infection surveillance on Methicillin-resistant Staphylococcus aureus. J Hosp Infect. 2005; 60: 172-175

${ }^{6}$ Wood MJ. The comparative efficacy and safety of teicoplanin and vancomycin. $J$ Antimicrobial Chemother. 1996; 37: 209-222.

${ }^{7}$ Smith TL, Pearson ML, Wilcox KR, et al. Emergence of vancomycin resistance in Staphylococcus aureus. Glycopeptide-Intermediate Staphylococcus aureus Working Group. N Engl J Med. 1999; 340: 493-501.

8 Song JH, Hiramatsu K, Suh JY, et al. Emergence in asian countries of Staphylococcus aureus with reduced susceptibility to vancomycin. Antimicrob Agents Chemother. 2004; 
48: 4926-4928.

9 Pea F, Brollo L, Viale P, et al. Teicoplanin therapeutic drug monitoring in critically ill patients: a retrospective study emphasizing the importance of a loading dose. $J$ Antimicrobial Chemother. 2003; 51: 971-975.

10 Garraffo R, Dellamonica P, Drugeon HB, et al. New approach to optimal antibiotic dosage regimen by coupling pharmacokinetics and killing curve parameters. Meth Finf Exp Clin Pharmacol. 1990; 12: 79-83

${ }^{11}$ Harding I, MacGowan AP, White LO, et al. Teicoplanin therapy for Staphylococcus aureus septicaemia: relationship between pre-dose serum concentration and outcome. $J$ Antimicrob Chemother 2000; 45: 835-41

${ }^{12}$ Derbyshire N, Webb DB, Roberts D, et al. Pharmacokinetics of teicoplanin in subjects with varying degrees of renal function. J Antimicrob Chemother 1989; 23: 869-876.

${ }^{13}$ Hillaire-Buys D, Peyriere H, Lobjoie E, et al. Influence of arterio-venous haemofiltration on teicoplanin elimination. Br J Clin Pharmacol. 1995; 40: 95-97.

${ }^{14}$ Rosina R, Villa G, Cavenaghi L, et al. Pharmacokinetics of teicoplanin in the elderly. J Antimicrob Chemother. 1988; 21: 39-45.

${ }^{15}$ Lortholary O, Tod M, Rizzo N, et al. Population pharmacokinetics study of teicoplanin in severely neutropenic patients. Antimicrob Agents Chemother. 1996; 40: 1242-1247. 
${ }_{16}$ Pirlich M, Schütz T, Kemps M, et al. Prevalance of malnutrition in hospitalized medical patients: impact of underlying disease. Digestive Dis. 2003; 21: 245-251.

${ }^{17}$ Cupisti A, D’Alessandro C, Morelli E, et al. Nutrition status and dietary manipulation in predialysis chronic renal failure patients. J Renal Nutr. 2004; 14: 127-133.

${ }^{18}$ Bailey EM, Rybak M, and Kaatz GW. Comparative effect of protein binding on the killing activities of teicoplanin and vancomycin. Antimicrob Agents Chemother.1991; 35: 1089-92.

${ }^{19}$ Bernareggi A, Borgonovi M, del Favero A, Rosina R, Gavanaghi L. teicoplanin binding in plasma following administration of increasing intravenous doses to healthy volunteers. Eur J Drug Metab Pharmacokinet 1991; Spec. 3: 256-60

${ }^{20}$ Pinnell AE, Northam BE. New automated dye-binding method for serum albumin determination with bromcresol purple. Clin Chem 1978; 24: 80-6

${ }^{21}$ Bland JM, Altman DG. Statistical methods for assessing agreement between two methods of clinical measurement. Lancet 1986; i 307-10.

${ }^{22}$ Mimoz O, Rolland D, Adoun M, et al. Steady-state trough serum and epithelial lining fluid concentrations of teicoplanin $12 \mathrm{mg} / \mathrm{kg}$ per day in patients with ventilator-associated pneumonia. Intensive Care Med 2006; 32:775-9

${ }^{23}$ Mabuchi H, Nakanishi H. Isolation and characterization of an endogenous drug-binding inhibitor present in uremic serum. Nephron 1986; 44: 277-81 
${ }^{24}$ Mabuchi H, Nakahashi H. A major inhibitor of phenytoin binding to serum protein in uremia. Nephron 1988; 48: 310-4.

\section{Figure Legends}

Figure 1 The effects of serum albumin levels on the fraction unbound of teicoplanin.

Symbols represent the measured fraction unbound of teicoplanin for all patients, and

the line represents the relationship predicted by the model described in figure 2 .

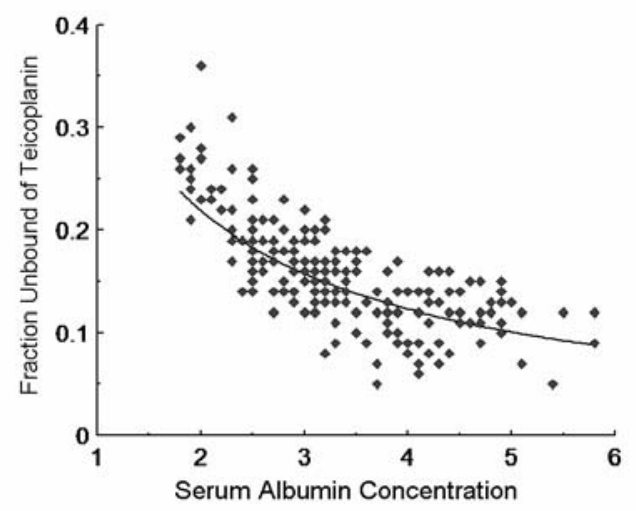

(g/dL) 
Figure 2 Three-dimensional plot showing the relationship between the concentration of free serum teicoplanin as a function of the concentration of total serum teicoplanin and serum albumin concentrations in the model developing data set.

Free serum teicoplanin concentrations were fitted by the least squares regression using the model presented in this figure, where $\boldsymbol{n}$ is the number of drug binding sites per albumin molecule with their association constant $(\mathrm{Ka}) . \mathrm{C} f$ and $\mathrm{Ct}$ mean free and total serum teicoplanin concentrations, respectively. ALB represents serum albumin concentrations. The curved surface represents the model fit based on linear regression analysis.

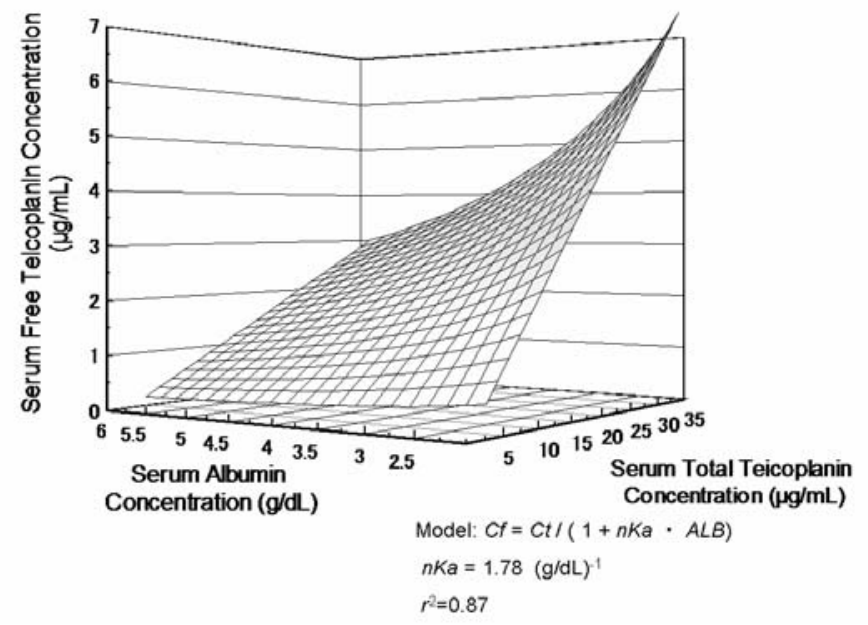

Figure 3 Difference plot of measured and predicted free serum teicoplanin concentrations in the model validation data set after logarithmic transformation.

The solid line denotes the mean difference and dashed lines denote upper and lower limits of agreement. SD means standard deviation 


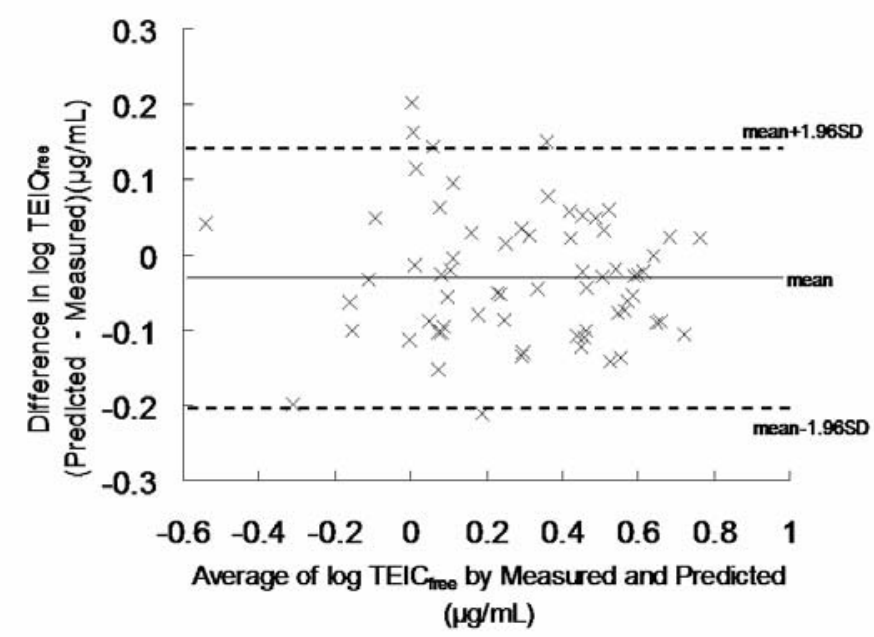

Figure 4 Nomogram to predict the free serum teicoplanin concentration based on the total teicoplanin concentration in patients with various serum albumin levels.

To estimate the free serum teicoplanin concentration, locate the point on the chart where the total serum teicoplanin concentration and the serum albumin intersect. Read the value of this point on the Y-axis.

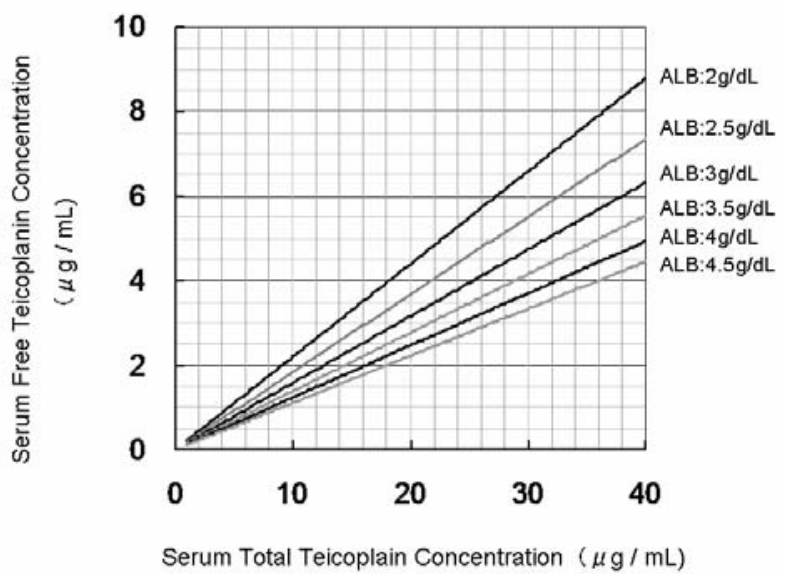

